\title{
Influence of plasma condition on carbon nanotube growth by rf-PECVD
}

\author{
Y. H. Man, Z. C. Li and Z. J. Zhang*
}

Carbon nanotubes (CNTs) have been synthesized from $\mathrm{Ar}-\mathrm{CH}_{4}$ mixtures using rf-plasma enhanced chemical vapor deposition (rf-PECVD) at $500^{\circ} \mathrm{C}$. Reduction gases such as $\mathrm{H}_{2}$ and $\mathrm{NH}_{3}$ were found unnecessary for carbon nanotube formation compared to thermal CVD. The relationship between the growth of CNTs and the plasma condition in PECVD has been investigated by in situ self bias measurement. Plasma conditions were controlled by changing the interelectrode distance, rf power and the applied substrate negative bias. By increasing the interelectrode distance and rf power, the spatial density of CNTs was on a rise as a result of the increase in ions density and self bias. As the applied substrate negative bias increased, the spatial density of CNTs decreased possibly due to the positive ions over bombarding effect.

Keywords: Carbon nanotube; Plasma enhanced CVD; Plasma condition

Citation: Y. H. Man, Z. C. Li and Z. J. Zhang, "Influence of plasma condition on carbon nanotube growth by rf-PECVD", Nano-Micro Lett. 2, 37-41 (2010). doi: 10.5101/nml.v2i1.p37-41

Plasma enhanced CVD (PECVD) has been recognized as one of the viable fabrication techniques of carbon nanotubes (CNTs) to produce vertically aligned nanotubes on patterned substrates at relatively low temperature [1-4]. A variety of plasma reactors have been used to grow carbon nanotubes. According to the types of plasma source to generate the gas discharge there are three main plasma reactors. One is direct current plasma CVD with a bias voltage of several hundred volts imposing on the cathode (sample) to initiate the dc discharge between the cathode (sample) and the anode [1-2,5-7]. Another common plasma reactor is microwave plasma CVD which uses a 2.45 $\mathrm{GHz}$ microwave generator to supply power for the plasma [8-11]. Radio frequency plasma CVD, divided into inductively coupled plasmas (ICPs) and capacitively coupled plasmas, also provides high-density plasma source with $13.56 \mathrm{MHz}$ power supply for CNTs growth [3-4,12-27]. The types of plasma reactor greatly affected the plasma condition and thus the growth environment for CNTs.

It has been revealed that the CNT growth was strongly influenced by the plasma condition. The plasma condition could be controlled through varying the react pressure, temperature, plasma power and deposition $\left(\mathrm{CH}_{4}, \mathrm{C}_{2} \mathrm{H}_{4}, \mathrm{C}_{2} \mathrm{H}_{2}\right)$ to etching $\left(\mathrm{NH}_{3}, \mathrm{H}_{2}\right)$ gas flow ratios, to control the crystallinity and the surface morphology of carbon nanotubes [1,5,9,14-15,19,21].
Besides, by the application of dc bias to the substrate during the growth, the alignment and spatial density of CNTs have a significant change [10,12]. Furthermore, the plasma condition could also be changed by varying the interelectrode distance in PECVD resulting in the variation of diameter and density of CNTs [27]. However, how the plasma condition exactly affects the growth of CNTs is not clear. There have been a few mentions on the plasma diagnostics to enhance our understanding of growth mechanisms. Luckily, several groups have investigated the influence of rf self-bias on the surface morphology and structure of diamond-like carbon (DLC) film during PECVD [28-30]. In this work, we attempted to use the self-bias to evaluate the plasma condition and further to illustrate the relationship between the growth of CNTs and plasma conditions.

The present paper focuses on the influence of the plasma condition on the surface morphology of CNTs using in situ self-bias plasma diagnostics. CNTs were grown at the temperature lower than the glass softening temperature (about $550^{\circ} \mathrm{C}$ ) without additive gases such as $\mathrm{NH}_{3}$ and $\mathrm{H}_{2}$ by capacitively coupled rf-PECVD system. Plasma condition was controlled by modulating rf power, interelectrode distance and the negative bias imposed on the substrate. The correlation between the growth morphology and the plasma condition is 
discussed.

\section{Experimental details}

Carbon nanotube was synthesized in a homemade rfPECVD reactor, as shown in Fig.1 (a). The reactor consists of two equal parallel plate electrodes, with diameter of $80 \mathrm{~mm}$, housed inside a high vacuum stainless steel chamber. A gas showerhead at the upper electrode was utilized to keep the growth of CNTs uniform. The upper electrode is connected to a 13.56 $\mathrm{MHz}$ power supply capacitively coupled through a matching network and the lower electrode simultaneously holding the substrate is grounded. A negative dc bias ranging from 0 to $-100 \mathrm{~V}$ is applied to the lower electrode. The interelectrode distance can be varied from 1 to $8 \mathrm{~cm}$.

$17 \mathrm{~nm}$ thick $\mathrm{Al}$ was deposited as the buffer layer between Fe catalyst layer ( $3 \mathrm{~nm}$ thick) and $\mathrm{Si}$ wafer by electron-beam evaporation at room temperature. The process for the growth of CNTs consists of three steps: (1) heat treatment; (2) Ar plasma pretreatment and (3) Ar- $\mathrm{CH}_{4}$ plasma treatment. After the rfPECVD reactor chamber was evacuated to a base pressure of about $10^{-5} \mathrm{~Pa}$, substrate was heated in a vacuum atmosphere without any reductive gases. Ar gas was then introduced into the chamber and maintained at 0.9 Torr with a flow rate of 100 sccm when the synthesis temperature reached $500^{\circ} \mathrm{C}$. At the second step, Ar plasma was introduced at $30 \mathrm{~W}$ for $5 \mathrm{~min}$ to pretreat the catalyst. $\mathrm{CH}_{4}$ gas was then introduced into the chamber and kept at 1 Torr, initiating the synthesis process. After 20 min synthesis, the sample was cooled down by mechanical pump. At the third step, various $\mathrm{rf}$ power $(30,50$, $70,100$ and $150 \mathrm{~W})$ and $\mathrm{CH}_{4} / \mathrm{Ar}$ ratio $(1: 0,1: 2,1: 5)$ were employed for the parametric study.

During the whole PECVD process, in situ plasma diagnostics were carried out. The rf power produces a plasma between the electrodes, as shown in Fig. 1(b). The higher mobility of the electrons than that of the ions in the plasma creates a sheath next to the electrodes with an excess of ions. Thus the plasma develops a positive voltage with respect to the electrodes [31]. The sheaths act as diodes, so that the electrodes acquire dc self-bias voltages equal to their peak rf voltage [32]. The self-bias was measured by a voltmeter (equivalent circuit was shown in Fig. 1(a)). The surface morphology of CNTs under different plasma conditions was characterized by scanning electron microscopy (SEM).

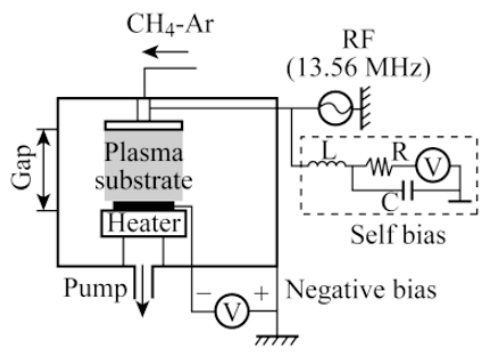

(a)

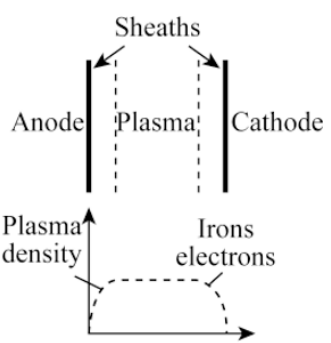

(b)
FIG. 1. (a) Schematic diagram of the rf-PECVD system with a plasma diagnostic apparatus for self-bias measurement and additional negetive bias applied to the substrate; (b) Electron and ion distributions which creat sheaths between the neutral plasma and the electrodes.

\section{Results and discussion}

$\mathrm{H}_{2}$ and $\mathrm{NH}_{3}$ were often used during thermal CVD and PECVD to reduce the catalyst and etching by-products such as amorphous carbon. In our work, we attempted to synthesize CNTs from Ar- $\mathrm{CH}_{4}$ mixtures without adding $\mathrm{H}_{2}$ and $\mathrm{NH}_{3}$. Both thermal CVD and rf PECVD were applied to synthesize CNTs for comparison. Figure 2(a) and 2(c) show the surface morphology of Fe film without and with pretreatment under Ar plasma for 5 minute before CNTs growth. The Fe film was transformed into more uniformly agglomerated small particles compared to the Fe film annealed without pretreatment. As is shown in Fig. 2(b), large amounts of particles with sporadic carbon nanotubes were deposited on the substrate by thermal CVD. From the inset of Fig. 2(b), energy dispersive X-ray spectrum (EDS) analysis showed that the intensity of carbon element was larger than the iron indicating the particles were carbon. In contrast, under rf PECVD CNTs were grown uniformly on the substrate with the length about $1 \mu \mathrm{m}$ and outer diameter of $20 \mathrm{~nm}$ without any amorphous carbon. It was found that reduction gases such as $\mathrm{H}_{2}$ and $\mathrm{NH}_{3}$ were not necessary for carbon nanotube formation during PECVD compared to thermal CVD. In thermal CVD, $\mathrm{CH}_{4}$ does not dissociate in the gas phase and the nanotube production is entirely due to the surface reaction of $\mathrm{CH}_{4}$ on the catalyst surface. With no $\mathrm{H}_{2}$ or $\mathrm{NH}_{3}$ added, catalyst particles would keep oxidation or be poisoned due to the deposition of amorphous carbon, resulting in a poor growth of CNTs. In contrast, plasma reactors produce, through electron impact as well as neutral reactions, great amounts of $\mathrm{C}_{\mathrm{x}} \mathrm{H}_{\mathrm{y}}$ radicals and ions from the methane feedstock, all of which contribute to the nanotube production [33].
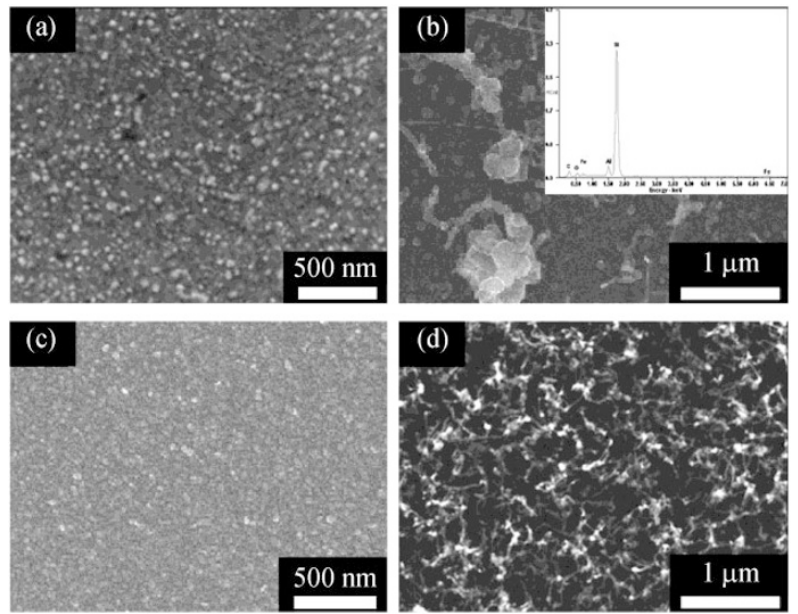

FIG. 2. (a) and (c) corresponding to the SEM morphology of catalyst film without and with pretreatment under Ar plasma for 5 minute before CNTs growth; (b) and (d) was the SEM morphology of CNTs grown by thermal CVD at $900^{\circ} \mathrm{C}$ and by rf-PECVD at $500^{\circ} \mathrm{C}$, respectively.

CNTs were synthesized from $\mathrm{Ar}-\mathrm{CH}_{4}$ mixtures using rf-PECVD under different growth environment. The growth morphology of CNTs and plasma condition were investigated as a function of the interelectrode distance, the rf power and the applied substrate bias.

First, the impact of plasma power on the growth of CNTs was investigated. Plasma power is a variable that can significantly alter the growth environment. Here, the CNTs 
were prepared at the interelectrode distance of $6 \mathrm{~cm}$ with $\mathrm{CH}_{4} / \mathrm{Ar}$ ratio of 1:0. Plasma power was varied from 30 to 100 W. As is demonstrated from Fig. 3(a), (b) and (c), with the moderately increase in the rf power, the length of CNT remain almost unchanged while the spatial density showed a significantly increase. Moreover, by increasing the power input the purity of CNTs had a great improvement. A large amount of carbon particles were deposited on the substrate when the rf power was $30 \mathrm{~W}$. At $70 \mathrm{~W}$, scarce CNTs with fewer carbon particles can be seen. Further increasing the power input, carbon nanotubes become cleaner. Thus, the rf power greatly influences the spatial density and purity of CNTs. Figure 3(d) illustrated the relationship between $\mathrm{rf}$ power and self bias. The $\mathrm{dc}$ self bias rises gradually with the rf power increased. The bias voltage $\left(\mathrm{V}_{\mathrm{b}}\right)$ for a resistive plasma will vary roughly with $\mathrm{rf}$ power (W) and pressure (P) as [34]

$$
\mathrm{V}_{\mathrm{b}}=\mathrm{k}^{\prime} \frac{\mathrm{W}}{\mathrm{P}^{1 / 2}}
$$
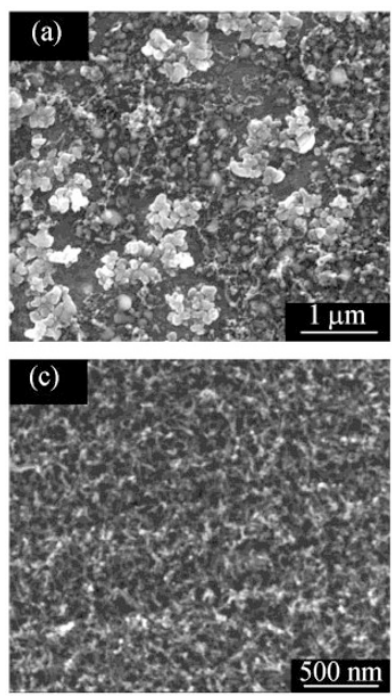

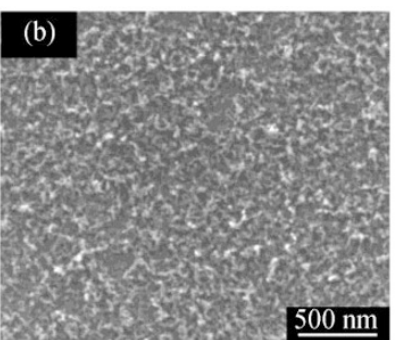

(d)

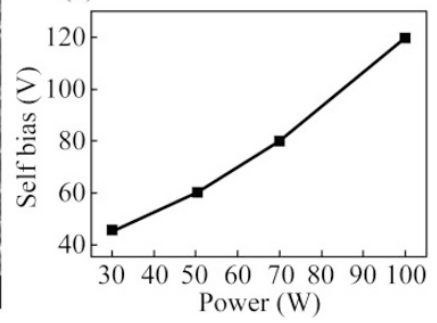

FIG. 3. SEM images of the CNT grown at the rf power of (a) $30 \mathrm{~W}$, (b) $70 \mathrm{~W}$ and (c) $100 \mathrm{~W}$, (d) self bias versus rf power.

In this section, the pressure was kept as a constant. Therefor, as the $\mathrm{rf}$ power increased, the self bias also increased. And the sheath voltage accelerates the positive ions to give the bombardment needed to create hydrocarbon radicals for the CNTs growth.

Next, we investigated the effect of the interelectrode distance on the growth of CNTs. The CNTs were synthesized at rf power of $150 \mathrm{~W}$ with $\mathrm{CH}_{4} / \mathrm{Ar}$ ratio of 1:2. Figure 4(b), (c) and (d) shows the surface morphology of CNTs synthesized at the interelectrode distance of $2 \mathrm{~cm}, 4 \mathrm{~cm}$ and $6 \mathrm{~cm}$, respectively. At $2 \mathrm{~cm}$, very short (less than $100 \mathrm{~nm}$ long) and sporadic CNTs were grown on the substrate with a small amount of amorphous carbon particles (which was also confirmed by EDS). When the interelectrode distance increased to $4 \mathrm{~cm}$, sparse CNTs can be seen without amorphous carbon. The average outer diameter and length of CNTs were about $20 \mathrm{~nm}$ and $500 \mathrm{~nm}$. Further increasing the interelectrode distance to $6 \mathrm{~cm}$, the spatial density of CNTs increases and the length up to $1 \mu \mathrm{m}$. Thus, the interelectrode distance strongly influences the morphology of CNTs, which is likely due to the change in the plasma condition. Self bias generated due to the difference in mobility between ions and electrons in plasma was in situ measured by a voltmeter as a function of the interelectrode distance. Figure 4(a) revealed dependences of interelectrode distance on self bias. It was found that self bias rises gradually with the interelectrode distance increased. In the dc-PECVD system with two equal parallel plate electrodes, the operating pressure (p) and the electrode gap (d) are constrained by the relation $\mathrm{p} \times \mathrm{d}=$ constant [35]. However, there is no quantitative statement about the relationship between $\mathrm{p}$ and $\mathrm{d}$ with respect to rf-PECVD. We observed that, with the electrode distance increases, there is a slight reduction in the pressure during the rf-PECVD process. According to formula (1), with the increase of the interelectrode distance, the pressure decreased slightly resulting in a small increase in the self bias. The sheath voltage accelerated the movement of the positive ions to the substrate. Among them, hydrocarbon radicals and ions benefit the CNTs growth. Therefore, with the interelectrode distance increased, higher density ions and hydrocarbon radicals are generated which contribute to the CNTs growth.
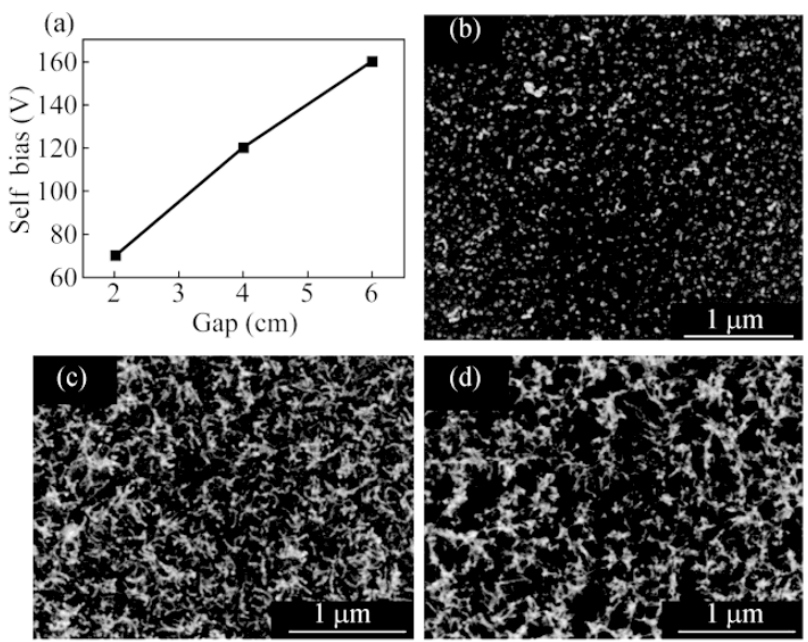

FIG. 4. (a) Dependences of interelectrode distance on self bias; SEM images of the CNT synthesized at the interelectrode distance of (b) $2 \mathrm{~cm}$, (c) $4 \mathrm{~cm}$ and (d) $6 \mathrm{~cm}$.

Finally, we investigated the influence of the applied substrate negative bias on the growth of CNTs. CNTs were grown at the interelectrode distance of $6 \mathrm{~cm}$ with $\mathrm{CH}_{4} / \mathrm{Ar}$ ratio of 1:5 and rf power of $50 \mathrm{~W}$. The applied substrate bias varied from 0 to $-80 \mathrm{~V}$. Figure 5(b), (c) and (d) shows the SEM images of CNTs synthesized at the applied substrate bias of $0,-20$ and $-40 \mathrm{~V}$, respectively. When the substrate is grounded $(0 \mathrm{~V})$, high-spatial density CNTs were grown on the substrate. After the substrate was imposed on a bias of $-20 \mathrm{~V}$, SEM characterization shows sporadic nanotubes grown on the substrate. Compared to the sample obtained at $-20 \mathrm{~V}$, the spatial density of CNTs grown at $-40 \mathrm{~V}$ was much lower. The spatial density of CNTs demonstrated a strong dependence on the magnitude of the applied bias. Figure 5(a) shows the variations of self bias with applied substrate negative bias. It was found that the self bias rose slightly from $100 \mathrm{~V}$ to $122 \mathrm{~V}$ as the negative bias increased from $0 \mathrm{~V}$ to $-80 \mathrm{~V}$. The voltage applied on the substrate can be controlled to modulate both ion energy and plasma species density. By increasing the applied substrate negative bias, positively charged ions such as $\mathrm{Ar}^{+}$and $\mathrm{H}^{+}$are accelerated to the substrate resulting in the bombardment. The 
sheath voltage and the applied substrate negative bias together possibly over-accelerate the positive ions to the substrate and thwart the CNT growth. The dc biasing appeared to have a strong effect on the growth of CNTs even though more systematic studies are required to comfirm the effect of dc biasing on the self bias and sheath voltage during the growth of CNTs using PECVD.
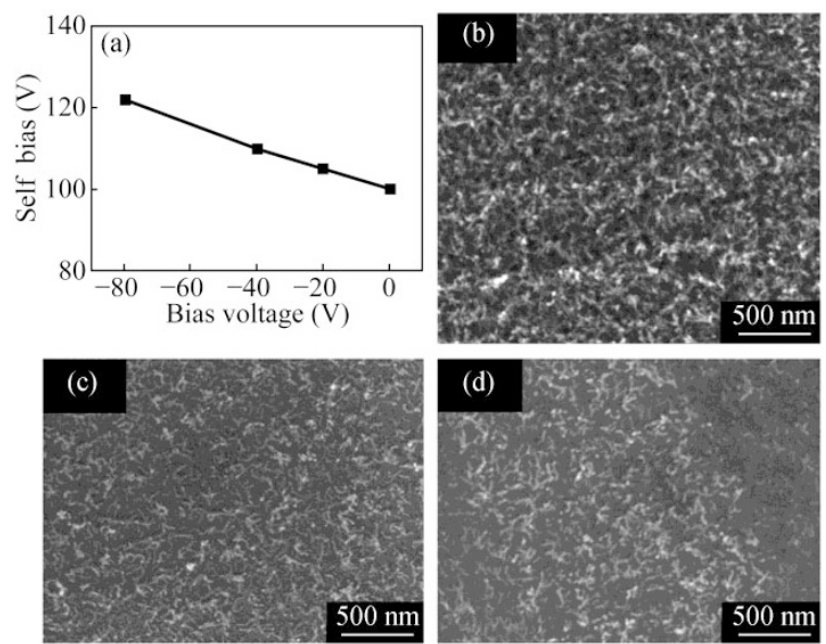

FIG. 5. (a) Variations of self bias with the applied substrate negative bias; SEM images of the CNT synthesized at the negative bias of (b) $0 \mathrm{~V}$, (c)-20 V and (d) $-40 \mathrm{~V}$.

The entire height of the growing CNTs is submerged inside the sheath. Plasma conditions strongly influenced the sheath voltage and the growth environment of CNTs. When the plasma condition varied as a function of the interelectrode distance, the rf power and the applied substrate bias, the self bias and sheath voltage also changed. When the interelectrode distance and the rf power increased, the voltage of self bias rose siginificantly. By increasing the applied substrate bias the voltage of self bias also showed a gentle rise. On the other hand, the interelectrode distance, the rf power and the applied substrate bias had a strong influence on the morphology of CNTs. That is to say, plasma condition greatly influenced the carbon nanotube growth during rf-PECVD. Through self bias we illustrated the relationship between the plasma condition and the growth of CNTs.

\section{Conclusions}

CNTs have been synthesized from $\mathrm{Ar}-\mathrm{CH}_{4}$ mixtures using rf-PECVD. Reduction gases such as $\mathrm{H}_{2}$ and $\mathrm{NH}_{3}$ were found unnecessary for carbon nanotube formation during PECVD because plasma reactors produce a large amount of $\mathrm{C}_{\mathrm{x}} \mathrm{H}_{\mathrm{y}}$ radicals and ions from the $\mathrm{CH}_{4}$ feedstock, which contribute to the nanotube production. Especially, the effects of the plasma condition on the growth morphology of CNTs have been investigated as a function of the interelectrode distance, the plasma power and the applied substrate bias, combining with the self bias measurement. The relationship between the plasma condition and the growth of CNTs would be favorable for further well-controlled aligned carbon nanotubes growth.

The authors are grateful to the financial support by the National Natural Science Foundation of China (grant No: 10675070,
50701026), and the National Basic Research Program of China (973 program, 2007CB936601).

Received 3 March 2010; accepted 19 March 2010; published online 29 March 2010.

\section{References}

1. C. S. Cojocaru, D. Kim, D. Pribat and J. Bourée, Thin Solid Films 501, 227 (2006). doi:10.1016/j.tsf.2005.07. 162

2. K. H. Jung, Y. Shin, J. Boo, Y. Kim and B. Hong, Thin Solid Films 501, 238 (2006). doi:10.1016/j.tsf.2005. $\underline{07.159}$

3. H. Wang, J. Lin, C. H. A. Huan, P. Dong, J. He, S. H. Tang, W. K. Eng and T. L. J. Thong, Appl. Sur. Sci. 181, 248 (2001). doi:10.1016/S0169-4332(01)00391-9

4. Z. F. Ren, Z. P. Huang, D. Z. Wang and J. G. Wen, Appl. Phys. Lett. 75, 1086 (1999). doi:10.1063/1.124605

5. M. Chhowalla, K. B. K. Teo, C. Ducati, N. L. Rupesinghe, G. A. J. Amaratunga, A. C. Ferrari, D. Roy, J. Robertson and W. I. Milne, J. Appl. Phys. 90, 5308 (2001). doi:10.1063/1.1410322

6. S. Hofmann, C. Ducati, J. Robertson and B. Kleinsorge, Appl. Phys. Lett. 83,135 (2003). doi:10.1063/1.1589187

7. D. H. Lee, W. J. Lee and S. O. Kim, Chem. Mater. 21, 1368 (2009). doi:10.1021/cm8034533

8. Y. Y. Wang, S. Gupta and R. J. Nemanich, Appl. Phys. Lett. 85, 2601 (2004). doi:10.1063/1.1796529

9. M. R. Maschmann, P. B. Amama, A. Goyal, Z. Iqbal, R. Gat and T. S. Fisher, Carbon 44, 10 (2006). doi:10.1016/ j.carbon.2005.07.027

10. M. R. Maschmann, P. B. Amama, A. Goyal, Z. Iqbal, R. Gat and T. S. Fisher, Carbon 44, 2758 (2006). doi:10.10 16/j.carbon.2006.03.040

11. C. Bower, O. Zhou, W. Zhu, D. J. Werder and S. Jin, Appl. Phys. Lett. 77, 2767 (2000). doi:10.1063/1.1319529

12. S. Kyung, Y. Lee, C. Kim, J. Lee and G. Yeom, Thin Solid Films 268, 506 (2006).

13. G. W. Ho, A. T. S. Wee, J. Lin and W. C. Tjiu, Thin Solid Films 388, 73 (2001). doi:10.1016/S0040-6090(01)00 $\underline{828-8}$

14. J. Han, W. Yang, J. Yoo and C. Park, J. Appl. Phys. 88, 7363 (2000). doi:10.1063/1.1322378

15. L. Valentini, J. M. Kenny, L. Lozzi and S. Santucci, J. Appl. Phys. 92, 6188 (2002). doi:10.1063/1.1515126

16. Y. Li, D. Mann, M. Rolandi, W. Kim, A. Ural, S. Hung, A. Javey, J. Cao, D. Wang, E. Yenilmez, Q. Wang, J. F. Gibbons, Y. Nishi and H. Dai, Nano Lett. 4, 317 (2004). doi:10.1021/n1035097c

17. U. J. Kim, E. H. Lee, J. M. Kim, Y. Min, E. Kim and W. Park, Nanotechnology 20, 295201 (2009). doi:10.1088/ 0957-4484/20/29/295201

18. T. Kato, G. Jeong, T. Hirata, R. Hatakeyama, K. Tohji and K. Motomiya, Chem. Phy. Lett. 381,422 (2003). doi:10. 1016/j.cplett.2003.10.007

19. A. Gohier, T. M. Minea, M. A. Djouadi and A. Granier, J. Appl. Phys. 101, 054317 (2007). doi:10.1063/1.2654647

20. Y. Shiratori, H. Hiraoka, Y. Takeuchi, S. Itoh and M. Yamamoto, Appl. Phys. Lett. 82, 2485 (2003). doi:10. 
$\underline{1063 / 1.1566803}$

21. T. Kato and R. Hatakeyama, Chem. Vap. Deposition 12, 345 (2006). doi:10.1002/cvde.200506451

22. H. Sato, T. Sakai, M. Matsubayashi, K. Hata, H. Miyake, K. Hiramatsu, A. Oshita and Y. Saito, Vacuum 80, 798 (2006). doi:10.1016/j.vacuum.2005.11.008

23. A. Gohier, T. M. Minea, A. M. Djouadi, A. Granier and M. Dubosc, Chem. Phy. Lett. 421,242 (2006). doi:10.1016/ j.cplett.2005.12.105

24. T. Kato, G. H. Jeong, T. Hirata and R. Hatakeyama, Thin Solid Films 457, 2 (2004). doi:10.1016/j.tsf.2003.12.002

25. Y. H. Wang, J. Lin, C. H. A. Huan and G. S. Chen, Appl. Phys. Lett. 79, 2767 (2000).

26. Y. Yabe, Y. Ohtake, T. Ishitobi, Y. Show, T. Izumi, and H. Yamauchi, Diamond Relat. Mater. 13, 1292 (2004). doi:10.1016/j.diamond.2003.11.067

27. T. Ikuno, S. Takahashi, K. Kamada, S. Ohkura, S. Honda, M. Katayama, T. Hirao and K. Oura, Surf. Rev. Lett. 10,
611 (2003). doi:10.1142/S0218625X03005505

28. A. Lousa and S. Gimeno. J. Vac. Sci. Technol. A 15, 62 (1997). doi:10.1116/1.580477

29. S. F. Yoon, H. Yang, J. Ahn and Q. Zhang, J. Elect. Mater. 27, 44 (1998). doi:10.1007/s11664-998-0336-4

30. L. Yang, Y. Xin, H. Xu, Y. Yu and Z. Ning. Plasma Sci. Tech. 12, 53 (2010). doi:10.1088/1009-0630/12/1/12

31. M. A. Lieberman and A. J. Lichtenberg. Principles of Plasma Discharges and Materials Processing (1994).

32. J. Robertson. Mater. Sci. Eng. R. 37, 129 (2002). doi:10.1016/S0927-796X(02)00005-0

33. D. B. Hash and M. Meyyappana, J. Appl. Phys. 93, 750 (2003). doi:10.1063/1.1525854

34. A. Bubenzer, B. Dischler, G. Brandt and P. Koidl, J. Appl. Phys. 54, 4590 (1983).

35. M. Meyyappan, J. Phys. D: Appl. Phys. 42, 213001 (2009). doi:10.1088/0022-3727/42/21/213001 